\title{
Design and Implementation of Microcontroller Based Mobility Aid for Visually Impaired People
}

\author{
Ezeofor J. Chukwunazo ${ }^{1}$, Georgewill M. Onengiye ${ }^{2}$ \\ ${ }^{1}$ Department of Electronic \& Computer Engineering, University of Port Harcourt, Rivers State, Nigeria \\ ${ }^{2}$ Ken Saro-Wiwa Polytechnic Bori, Rivers State, Nigeria
}

\begin{abstract}
This paper presents the design and implementation of Microcontroller Based Mobility aid for visually impaired people. In the world today, about 285 million people are visually impaired. Over the years visually impaired persons have difficulty to interact and feel their environment. They have little contact with their surroundings, and physical movement is a very big challenge to them because it can become tricky to distinguish where he/she is and how to get where he/she wants to go. To navigate to unknown places has to be with individual support. Majority of the blind people in the world are unemployed because of the type of jobs available to them are limited; their mobility opposes them from interacting with people and social activities. This has created very huge concern to people assisting them to walk. This research work aimed at designing mobility aid to the visually impaired persons in order to encourage them to move freely anywhere they want at any time without collision and assistance. The proposed low cost and light weight system is designed with microcontroller that processes signal and alerts the visually impaired person over any obstacle, water or dark areas through beeping sounds. The system consists of special detection sensors, AT89C52 microcontroller for receiving, processing and sending signals to the alarm system which finally alerts the user for prompt action. The system was designed, programmed with assembly language and tested for accuracy and checked by the visually impaired person.
\end{abstract}

Keywords: Ultrasonic sensor, AT89C52 Microcontroller, Mobility aid, visually impaired person, Alarm system

\section{Introduction}

Visual impairment, known as vision impairment or vision loss, is a decreased ability to see to a degree that causes problems not fixable by usual means, such as glasses. Some also include those who have a decreased ability to see because they do not have access to glasses or contact lenses. Visual impairment is often defined as a best corrected visual acuity of worse than either 20/40 or $20 / 60$. The term blindness is used for complete or nearly complete vision loss. Visual impairment may cause people difficulties with normal daily activities such as driving, reading, socializing, and walking. The most common causes of visual impairment globally are uncorrected refractive errors $(43 \%)$, cataracts (33\%), and glaucoma (2\%). Refractive errors include near sighted, far sighted, presbyopia, and astigmatism. Cataracts are the most common cause of blindness (https://en.wikipedia.org/wiki/Visual_impairment).

The human being receives information from the environment, $1 \%$ by taste, $1.5 \%$ by tactile sense, $3.5 \%$ by smell, $11 \%$ by hear and $83 \%$ by sight. Artificial vision is the most important part of human physiology as $83 \%$ of information human being gets from the environment is via sight [1].The statistics by the world health estimates that there are 285 billion people in the world with vision impairment, 39 billion are blind and 246 with low vision. The oldest and traditional mobility aids for persons with visual impairments are the walking stick also known as the white cane stick and guide dogs. The draw backs of these aids are range of motion and very little information conveyed with the rapid advance of modern technology. The guide cane is light and portable but its range is limited to its own size and is not usable for dynamic component. The guide dogs are still far from being affordable, around the price of a car and there average working time is limited, an average of 7years [2].

However in comparison with other technology many blind guidance systems use ultrasound because of its immunity to the environmental noise and its technology is relatively inexpensive and ultra sound emitters and detectors are small enough to be carried about without the need of complex circuitry. Apart from the conventional navigating system, a blind aid system can provide a new dimension of real time assistance and artificial vision along with dedicated obstacle detection circuitry [3].

Ultrasonic sensor is the proposed electric aid which senses the obstacle in its path by continuously transmitting the ultrasonic waves when an obstacle appears in its vicinity then the ultrasonic waves get reflected to the system immediately, the ultrasonic receiver senses these ultrasonic waves. This method supports the microcontroller to obtain information from ultrasonic waves and then it alerts the blind pedestrians through sound messages. The advantage of this proposed system is its sound based alerts for easy navigation which can assist a blind pedestrian to pass through a busy road. Moreover in [4], this system is an auditory guidance system for the visually impaired pedestrians, using ultrasonic to audio signal transformation. This system presents a concept to provide a smart electronic aid for blind people, the system is intended to provide an overall measures artificial vision and object detection. This research work focuses on obstacle detection, light detection and water detection in order to reduce navigation difficulties for visually impaired people. 


\section{International Journal of Science and Research (IJSR) \\ ISSN (Online): 2319-7064}

Index Copernicus Value (2013): 6.14 | Impact Factor (2015): 6.391

\section{Related Work}

\subsection{Intelligent Guide Stick}

In 2001, a study was carried out for developing an intelligent guide stick for the blind using an intelligent central processing unit (CPU) called MELDOG. (Sung Young and Kim 2001). This system uses an artificial intelligence which can sense the accurate position of obstacle using ultrasonic sensors and laser sensors in other to identify the position. The "map matching technique" was used with ultrasonic sensors which include a direct current motor (D.C motor) controller that connects to an encoder. When the wheels rotate 18 degree, the infrared sensor attached to both wheels will transmit the signal to the CPU in other to provide a location update. This is an accurate detecting system which can provide the user continuous update for detecting the obstacle with detection angle between 0 degree to18 degrees. However, this system is too expensive and complex in designing. It is heavy compared to other similar systems; the weight of the system is around $5.5 \mathrm{~kg}$ [9]

\subsection{Laser Cane}

Benjamin et al, 2011 developed a smart stick using laser sensors to detect the obstacles and down curbs. Obstacles detection was signalized by a high pitch beep using a microphone. The design of the laser cane is usually simple and intrusive. The cane can only detect obstacles but cannot provide cognitive and psychological support. There exists only beep sound that triggers any obstacles and there is no assistance to direct them.

\subsection{Smart Stick}

Jacio Jose, Muguel Farrajota, and Joae M.F Rodnigues, 2011 designed a smart stick prototype. It was small in size, cheap and easy wearable navigation aid. This blind stick functions by addressing the global navigation for guiding the user to some destination and local navigation for negotiating path, sidewalks and corridors even with avoidance of statue as well as moving obstacles. Rather they invented a stereo camera worn at the chest height; a portable computer in a shoulder strapped pouch or pocket and one earphone or small speaker. The system is inconspicuous, and with no hindrance while working with the cane. Also it does not interfere with normal sound in the surroundings [6]

\subsection{Electronic Long Cane}

Alejandro R, Garcia Rumorez and Renato Penseca Livramento, de Solvaetal, 2012 designed an assistive technology device called the electronic long cane to serve as a mobility aid for the blind and visually impaired people. The author implements the cane with an ergonomic design and an embedded electronic system which fits inside the handle of the traditional long cane, the system was designed using hap tic sensor to detect obstacles above the waistline. It works in such a way that when an obstacle is detected, the cane vibrates or makes a sound. However, this only detects obstacles above the waistline [1].

\subsection{GPS Artificial Vision Assistance}

In 2011, another system was done by Shruit Dumbhare and Prof. A. Sakhore. The system offered obstacle detection, artificial vision and a real assistance via GPS. This system works by using GPS artificial vision, obstacle detection and a voice circuit and works by putting a camera on the persons head, the camera use an algorithm to identify the highs and obstacle in front of the blind person. This system also contains ultrasonic sensors to detect the obstacle furthermore. The GPS enables the system to connect to the required destination which provides accuracy output to the user.

\subsection{Smart Cane}

Another study was done by Jayant, Pratik and Mita, 2012. They proposed a smart cane assisted mobility for the visually impaired and the system is based on normal ultrasonic sensor and ATMEL microcontroller. It operates with two rechargeable batteries of $7.4 \mathrm{v}$ each and can be recharged through USB cable or A.C adopter. The control unit is programmed using embedded c-language; once any obstacle is detected, vibration and buzzer will be triggered to warn the user. The system has the ability to cover a distance up to 3 meters and also can be folded in small pieces so that the user can carry it easily. Moreover, this system has only one direction detection coverage and is inaccurate in detecting obstacles [7].

\subsection{Voice Operated Outdoor Navigation System}

Voice operated outdoor navigation system for the visually impaired person was done by Samantha and Ravi, 2012. It uses a stick equipped with ultrasonic sensor, GPS and audio output system. The stick contains GPS which has S.D memory card that stores different locations. The user can set the location by voice and the GPS will guide the person to his or her destination. This system also provides the speed and the remaining distance to reach the destination. When the ultrasonic sensor detects an obstacle directly, the voice system will activate the caution voice. The system uses the ARM processor which has more memory space, so that the operating speed is high. Nevertheless, this system cannot operate indoors because of GPS system that gets signal from satellite. The accuracy of the GPS signal needs to be improved because it can be controlled within 5 meters radius only. Finally, the blind person needs to be trained intensively so that he or she will be able to use the system effectively [8]

\subsection{Pulse Echo Technique}

Another study in the same field uses the pulse echo technique by G. Gayathri, 2014 in order to provide a warning sound when obstacle is detected. This technique is used by the United States military for locating the sub marines. They use pulse of ultrasound range from $20 \mathrm{kHz}$ to $50 \mathrm{kHz}$ which hits the hard surface to generate echo pulses by calculating the difference between signal transmit time and signal receiving time, one can predict the distance between the user and the obstacle. This system is very sensitive in terms of detecting the obstacle. It has a detecting range up to 3 meters and a detecting angle of 0 to 45 degree. However, the system 


\section{International Journal of Science and Research (IJSR) \\ ISSN (Online): 2319-7064 \\ Index Copernicus Value (2013): 6.14 | Impact Factor (2015): 6.391}

requires more power to operate because of the transmitter and the receiver circuits. Therefore this system needs to be redesigned to operate with less power [5].

\section{Method}

The system is design following a simple methodology that breaks down each unit of the system and separately dealing with each part to avoid complexity and difficulty in troubleshooting. The system comprises of four major units (figure 1)

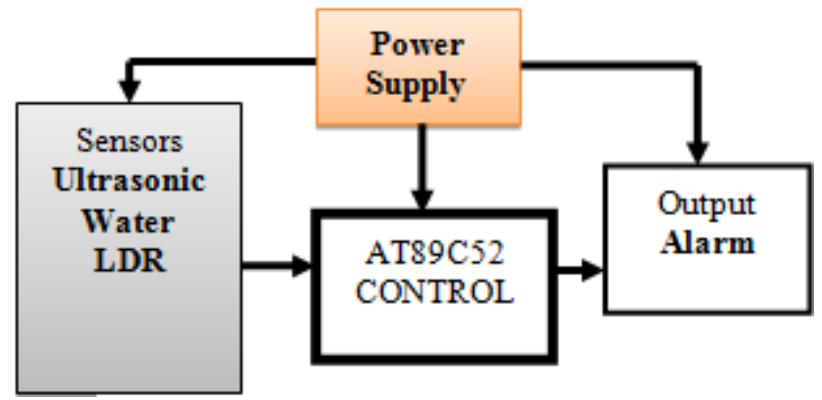

Figure 1: Smart Walking Stick block diagram

\subsection{Power Supply Unit}

The system is powered by a $9 \mathrm{~V}$ battery. The output noise from the battery source is filtered by a $100 \mathrm{uF}$ capacitor. Two voltage regulators (LM7805) are used to give $5 \mathrm{~V}$ fixed output for the microcontroller and the Alarm buzzer system. A $1 \mathrm{k} \Omega$ is used to limit current going through the LED power indicator. The circuit diagram of the power supply is shown in (figure 2).

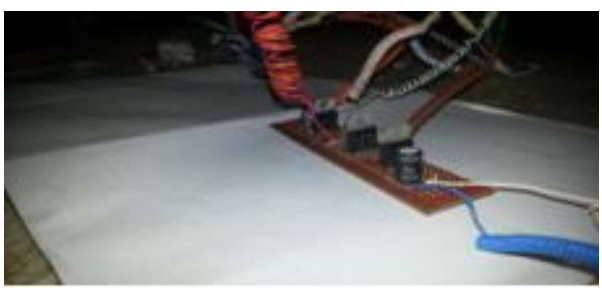

Figure 2: Power supply unit

\subsection{Sensor Input Unit}

The input unit comprises of an ultrasonic sensor (obstacle detector), light detection and water detection.

A. Ultrasonic sensor: The ultrasonic sensors are devices that transmit an electrical signal when come in contact with an obstacle, it transmit $5 \mathrm{~V}$ through the echo as in (figure 3).
B. Light Detection: The circuit is made up of a light dependent resistor (LDR), potential divider network as shown in (figure 4), transistor and comparator. The LDR is connected in series with a $100 \mathrm{k} \Omega$ resistor $\mathrm{R} 2$ to divide the voltage across the LDR. The output voltage from that connection (voltage drop across LDR) is sent to the pin 2 of the voltage comparator LM393 which is the inverting terminal. The potential divider network is between R3 and $\mathrm{R} 4$ the output from this connection VR4 is used as the reference voltage of the comparator. It is connected to pin 3 of LM393 voltage comparator which is the non- inverting terminal. The comparator compares two voltages between its input voltage terminals Vin Pin 2 (inverting) and its reference voltage Vref Pin 3 (non-inverting) and gives out a corresponding output.

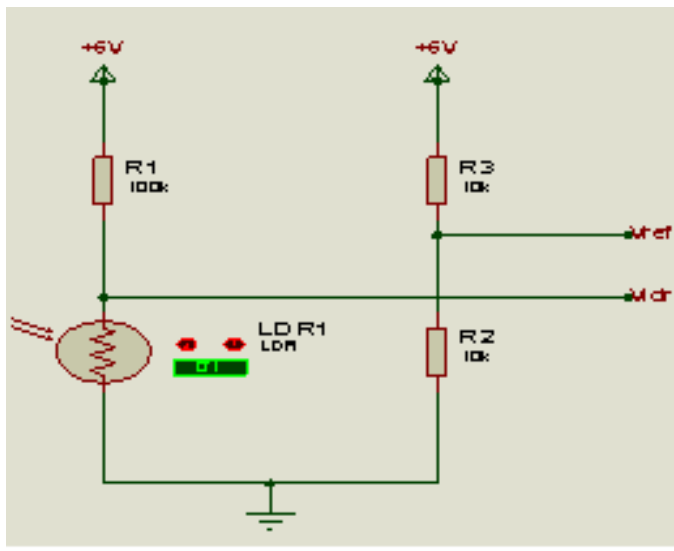

Figure 4: Potential Divider Network Circuit

The comparator output voltages are determined between the voltages at Pin 2 and Pin 3. If inverting terminal Vin $>$ Vref the output voltage will be a $5 \mathrm{~V}$. If the non - inverting terminal Vref > Vin the output voltage will be a $0 \mathrm{~V}$. This feature of the comparator is what we exploited to design the light detector. At night the LDR gives a very high resistance value ranging up to $2 \mathrm{M} \Omega$ and in the day time or when there is sun light it give a low resistance ranging to $100 \Omega$ and sometimes below. From the voltage divider network at day time the voltage from the LDR is lower there by making pin 2 lower than pin 3 of the comparator giving an output voltage of $0 \mathrm{~V}$ and at night the $\mathrm{V}_{\mathrm{LDR}}$ is high making pin 2 greater and the comparator output $5 \mathrm{~V}$. The output is fed to a potential divider biased transistor switch. The transistor network inverts this signal and sends it to the control unit. The output from the transistor is sent to P1.2 of the microcontroller which through a programmed gives out a corresponding output in the output unit. The light detection circuit is shown in (figure 5).

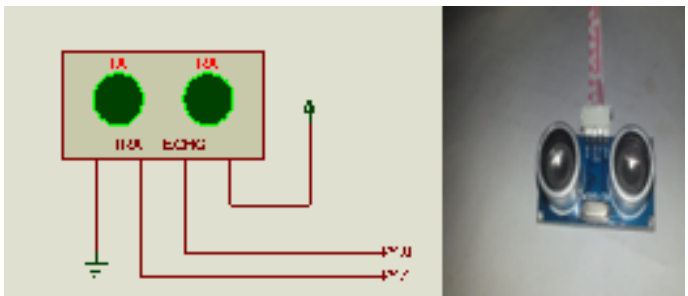

Figure 3: ultrasonic sensor 


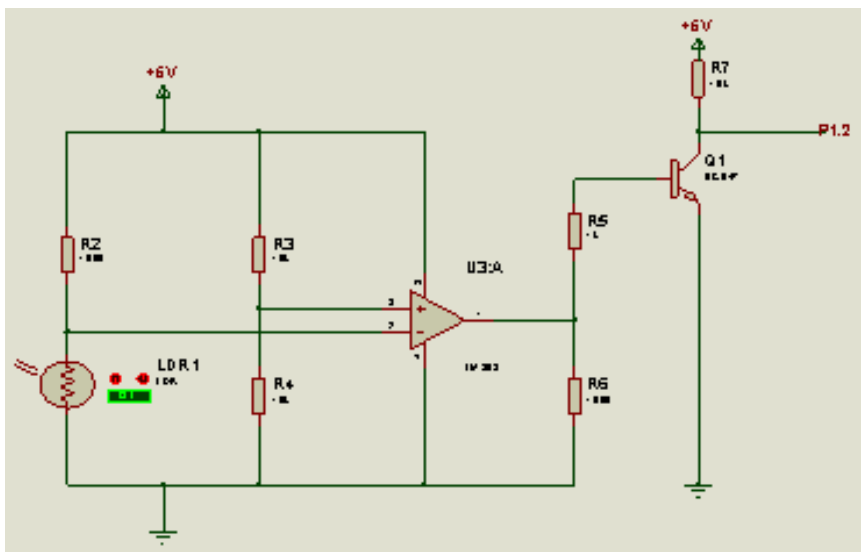

Figure 5: Light Detection Circuit

C. Water Detector: The water detector system uses a conductor system incorporated with an NPN transistor BC547 and a conducting wire. The water detector is shown in (figure 6). The conductor wire is connected to $5 \mathrm{~V}$ voltage source. Two other points are created to receive the $5 \mathrm{~V}$ from the water, knowing that water is a conductor. When the other probe conducts electricity from the water it forward biases a transistor and turns ON the transistor. These transistors are configured as switch and inverter. The transistors invert these signals and send a corresponding output to the control unit. Probe 1 is connected to $\mathrm{P} 2.0$ and probe 2 connected to P2.1 of the microcontroller.

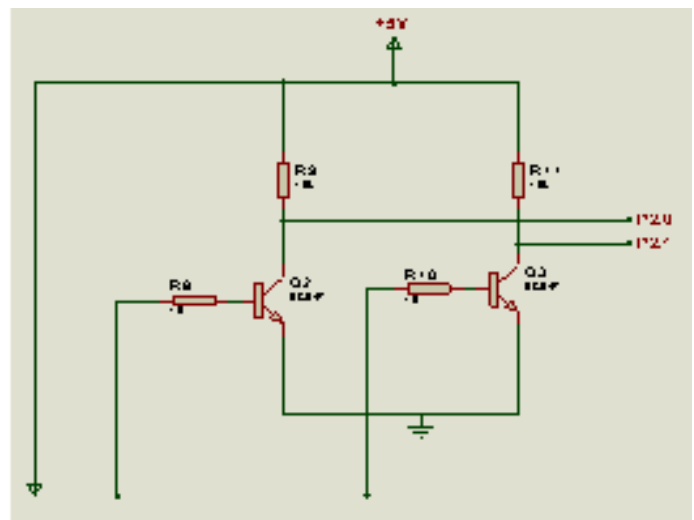

Figure 6: Water Detector Circuit

\subsection{AT89C52 Microcontroller Unit}

It is an 8-bit microcontroller and belongs to the Atmel 8051familily. AT89C52 has $8 \mathrm{kB}$ of flash programmable and erasable read only memory (PEROM) and 256 bytes of RAM (Random Access Memory). AT89C52 (figure 7) has an endurance of 1000 write/erase cycles which means that it can be erase and programmed to a move of 1000 times. The microcontroller consists of input and output ports which are used to receive and send out signals. The sensor outputs are fed into the input ports of the microcontroller. The microcontroller receives signals from the sensors, process and triggers the transistor to activate the buzzer ON which draws attention of the visual impaired person to act on.
AT89C52

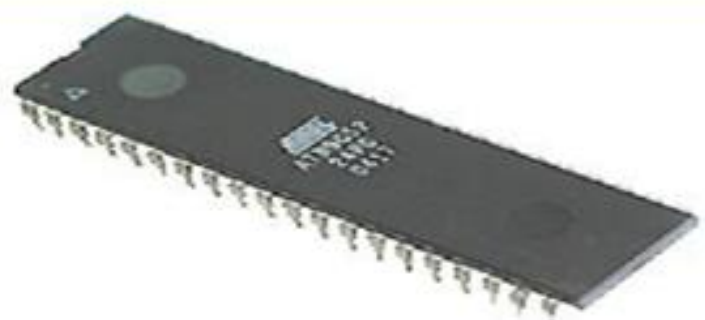

Figure 7: AT89C52 pictorial diagram

\subsection{The Output Unit (Buzzer)}

The output unit is made up of a transistor switch and a buzzer (alarm) as in (figure 8). The transistor switch is forward biased when the microcontroller receives data from any of the input sensor, process and sends it out on the ports connected with the transistor. If the signal comes from the ultrasonic sensor, it means that an obstacle is around the corner and the buzzer would sound trice to warn the user. Also if the signal is from the light detector, the buzzer would make a long sound and stops. Finally, if the signal is from the water detector, the buzzer would make a beep twice.

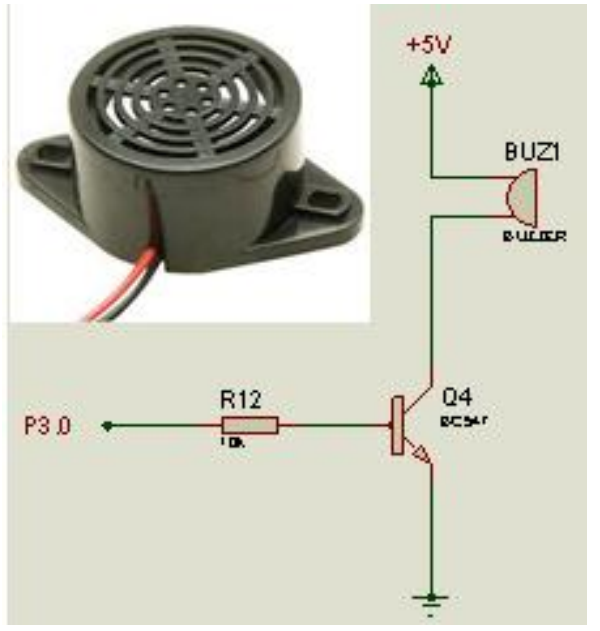

Figure 8: Buzzer circuit

\subsection{System Flow Chart}

The system algorithm comprises of three main steps (figure 9), step 1 boots the system on, step 2 scans for terminal, if the terminal is high (that is obstacle detected) it goes to step 3 to turn on the buzzer but if the terminal is low (no obstacle detected) it goes back to step 2 and scans for terminal.

1. Data terminal

2. Is any data high?

3. If yes turn Start

4. Scan sensors on the buzzer, then go to step 3

5. If no go to step 3 


\section{International Journal of Science and Research (IJSR) \\ ISSN (Online): 2319-7064}

Index Copernicus Value (2013): 6.14 $\mid$ Impact Factor (2015): 6.391

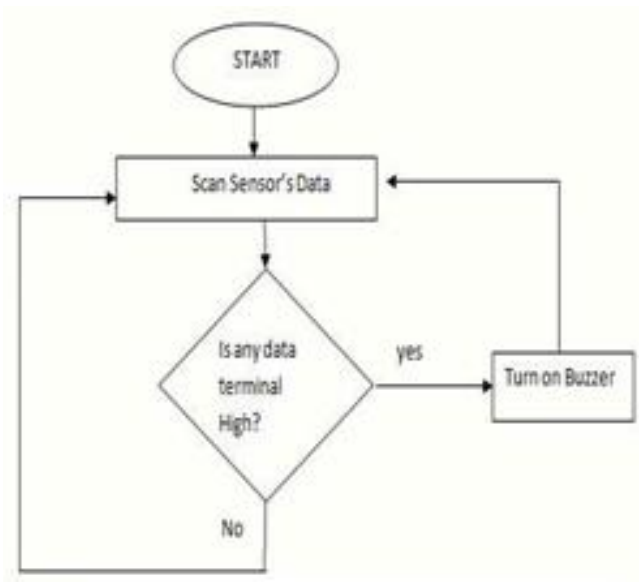

3.6 System Operation \& Circuit

When the blind person is gradually moving, the mobility aid will be held at his/her hand and drives in the direction of movement. The sensors attached on the body of the stick would be sensing obstacle, water and darkness.

Figure 9: Smart stick Algorithm

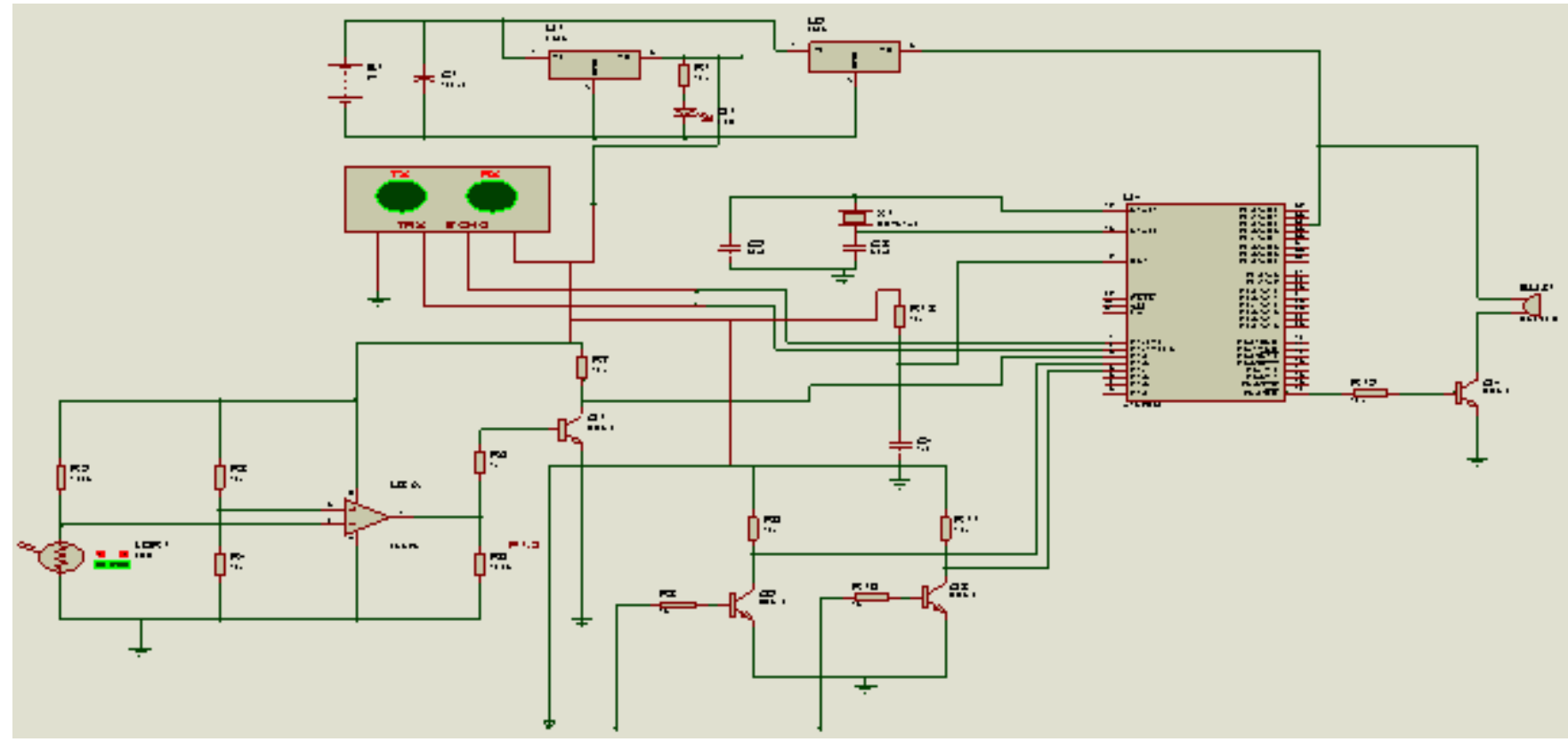

Figure 10: Mobility aid circuit diagram

As soon as any sensor detects something, it sends signal to microcontroller which processes the signal and triggers the transistor to activate the alarm/buzzer. The buzzer sound alerts the visual impaired person holding the smart stick for action to be taken to avoid collision. When action has been taken, the alarm will stop and the system continues monitoring and guiding. The smart stick circuit diagram is shown (figure 10) above.

\section{Test \& Result}

Testing and reliability check is an important aspect of design. Each of the components was tested for continuity and efficiency before soldering.

- LDR: The result for the light detection (LDR) worked as expected and the output voltages (Table 1) showed from the LDR is realistic. The alarm came ON at dark to warn the user its night or the place is dark.

- Ultrasonic Sensor: The result of the ultrasonic detector for the system worked as expected (Table 2) at a distance very close to the user. The buzzer came ON as expected warning the user that an obstacle is very close.
- Water Detection Sensor: (Table 3) is the result for water detection that aid the user not to deep his/her legs into stagnant water in the surrounding. If the walking aid touches any deep water, the transistors will trigger the alarm system to switch $\mathrm{ON}$.

Table 1: LDR Test Result

\begin{tabular}{|c|c|c|c|}
\hline $\begin{array}{c}\text { Time } \\
\text { (Hours) }\end{array}$ & $\begin{array}{c}\text { LDR }(\Omega) \\
\text { Resistance }\end{array}$ & $\begin{array}{c}\text { Voltage }(\mathrm{V}) \\
\text { across LDR }\end{array}$ & Alarm \\
\hline 7:00AM & 5338.35 & 0.1076 & Off \\
\hline 9:00AM & 829.78 & 0.0166 & Off \\
\hline 10:00AM & 728.44 & 0.0015 & Off \\
\hline 11:00AM & 730.00 & 0.0015 & Off \\
\hline 12:00PM & 600.34 & 0.0012 & Off \\
\hline 1:00PM & 500.32 & 0.0010 & Off \\
\hline 2:00PM & 456.40 & 0.0009 & Off \\
\hline 4:00PM & 300.45 & 0.0006 & Off \\
\hline 5:00PM & 9898.90 & 0.1900 & Off \\
\hline 6:00PM & 99342.23 & 1.9868 & Off \\
\hline 7:00PM & 150283.27 & 3.0006 & On \\
\hline
\end{tabular}




\section{International Journal of Science and Research (IJSR) \\ ISSN (Online): 2319-7064}

Index Copernicus Value (2013): 6.14 | Impact Factor (2015): 6.391

Table 2: Ultrasonic Sensor Test Result

\begin{tabular}{|r|c|c|}
\hline S/NO & Distance (Meters) & Alarm \\
\hline 1. & 0.6040 & On \\
\hline 2. & 0.8096 & Off \\
\hline 3. & 0.9144 & Off \\
\hline 4. & 1.2192 & Off \\
\hline 5. & 1.5240 & Off \\
\hline 6. & 1.8288 & Off \\
\hline 7. & 2.1336 & Off \\
\hline
\end{tabular}

Table 3: Water Detection Test Result

\begin{tabular}{|c|l|l|}
\hline Probes & No Water Detected & \multicolumn{1}{c|}{ Water Detected } \\
\hline Probe 1 1 & $\begin{array}{l}\text { 0V, the alarm is off. } \\
\text { The transistor is on } \\
\text { the off mode. }\end{array}$ & $\begin{array}{l}5 \mathrm{~V} \text {, the alarm came } \\
\text { ON. The transistor } \\
\text { is forward bias. }\end{array}$ \\
\hline Probe 2 & $\begin{array}{l}\text { OV, the alarm is off. } \\
\text { The transistor is on } \\
\text { the off mode. }\end{array}$ & $\begin{array}{l}\text { 5V. The alarm came } \\
\text { ON. The transistor } \\
\text { is forward bias. }\end{array}$ \\
\hline
\end{tabular}

\subsection{Hardware Implementation}

The system was designed and values of each component calculated and purchased. The implementation started with mounting of the components on the Vero board; each component was tested before mounting and then soldered to the Vero board. The power supply unit was built first and the voltages tested before the next stage. The control unit was soldered and tested as well. Several system units (figure 11) were assembled and tested for any fault before packaging with a plastic adaptable box. The final prototype (figure 12) shows mobility aid for the visually impaired persons.

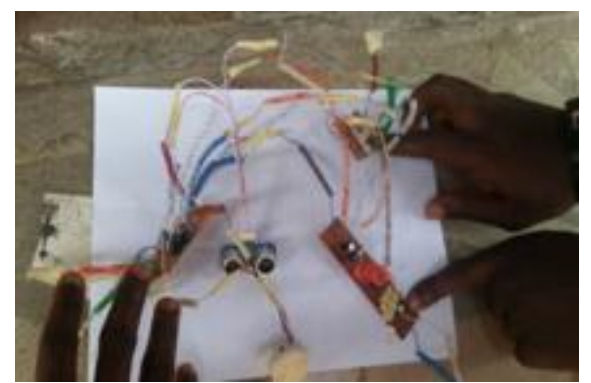

Figure 11: Hardware units of the system

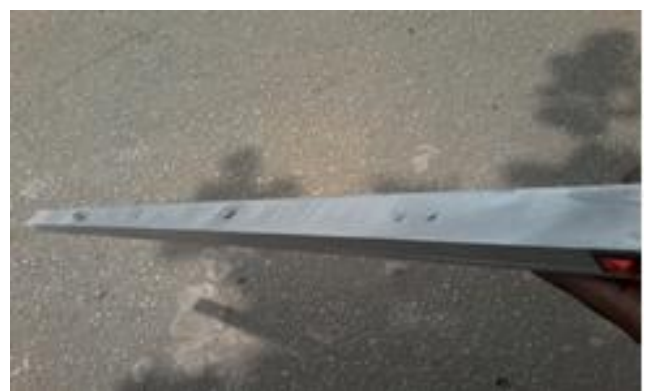

Figure 12: Implemented Mobility aid for the blind

\section{Conclusion}

The Mobility aid designed for blind people is affordable, reliable and easy to operate. It reduces stress to people assisting the blind and provides comfort to blind during walking. Various tests carried out and the results obtained would encourage the use of the system. The system worked accordingly to specification and quite satisfactory. The system is designed to gathers data from the environment via different sensors, processes the data and extracts viable information from the data, then transformed the information into an audio sound for the blind pedestrian to recognise and acts upon. The system consists of an ultrasonic sensor for obstacle detection, a water sensor for water detection and slippery areas and a light dependent resistor for dark detection. Each sensor is differentiated from one another through pattern of sounds.

\section{References}

[1] R Garcia Ramruz, Renato Punseca Luramento du Silva Milton Jose, Cinelli Alejandro Puran Carrilo de Albornoz, "Evaluation of electronic haptic device for blind and visually impaired people" Journal of medical and biological Engineering vol32.NO 6 423-428. 2012.

[2] Benjamin J.M, Alina, Scheps A.F , "A laser cane for the blind" proceedings of the san diego biomedical symposium vol.12, 53-57,2011,.

[3] Bousbia-Salah, M,M. Bettayeb and A. Larbi, "A navigation aid for blind people and of intelligent and Robotic systems" 64(3-4) P-387-400, 2011.

[4] Dambhara, S and Sakhara, A, "Smart stick for blind obstacle detection, Artificial vision and real time assistance via GPS" (International Journal of computers) 2011.

[5] G.Gayathri, "Smart walking stick for visually impaired" International Journal of engineering and computer sciences ,IJECS Volume 3, Issue 3, Page No 4057-4061, March 2014.

[6] Joao Jose, Miguel Farrajota, Joao M.F Rodrigues J.M Hans du Baf, "The smart vision local navigation aid for the blind and visually impaired persons" International journal of digital content and technology, Vol.5. 2011.

[7] Mounir Bousbia-Salah Abjelghani redjati, Mohamed Fezari, Muamar Bettyeb, "An ultrasonic navigation system for the blind people" Pricy 2007 IEEE International journal conference on signal processing and communications (ICSPC 2007) 24.27 November 2007, Dubai United Emirates.

[8] Shinohara K., " Designing assistive technology for blind users" In proceeding of the $8^{\text {th }}$ international ACMSIG access conference on computers and accessibility ACM 293-299. 2006.,

[9] Sungjae Kung Young Ho Kim and Inhyuk Moon, "Development of intelligent guide stick for the blind" IEEE International conference on robotics and automation seal, Korea 21, 26. 2001

\section{Author Profile}

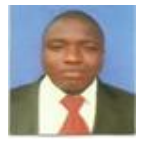

Ezeofor Chukwunazo Joseph received his B.Eng. in Computer Engineering at Enugu State University of Science and Technology (ESUT) and M.Eng in Communication Engineering at Federal University of Technology Owerri (FUTO). Ezeofor is currently studying Ph.D in Computer and Control Systems Engineering at Nnamdi Azikiwe University (NAU) Awka Nigeria. $\mathrm{He}$ has done over five publications and supervised more than fifteen students. He is a member Nigerian Society of Engineering (NSE) and registered member of Council of Regulation of Engineering in Nigeria

\section{Volume 5 Issue 6, June 2016 www.ijsr.net}


(COREN). His research interest is on Real Time Embedded Systems, Control Systems, and Telecommunication. He is happily married with children.

GEORGEWILL Onengiye Moses got his B.Sc. in Computer Science at University of Nigeria, Nsukka and first M.Sc in Applied Geophysics at Rivers State University of Science \& Technology Port Harcourt (RSUST).He also received his second M.Eng in Computer Engineering at the Federal University of Technology Owerri (FUTO). Georgewill further had third Master of Science degree, M.Sc in Computer Science at University of Port Harcourt (UNIPORT) and finally Ph.D in Computer Science in the same Institution. He is currently the Rector, Ken Saro Wiwa Polytechnic Rivers State, Nigeria. Georgewill has done over thirty publications. $\mathrm{He}$ is a member of Nigeria Computer Society (NCS), Association of Computer Machinery (ACM), Institute of Electrical and Electronic Engineering (IEEE), Computer Professionals (Registered Council) of Nigeria (CPN), International Research and Development Institute (IRDI). His research interest is in Artificial Intelligent (Fuzzy logic, Neural Network etc), Robotics, Computer Vision etc. He is happily married with children. 